\title{
Numerical calculation of the resistance of catamarans at different distances between two hulls
}

\author{
Zhaochun Liu ${ }^{1,}$, Xiufeng Zhang ${ }^{1, b^{*}}$, Yao Meng ${ }^{1, c}$, Linghong Wang ${ }^{1, d}$ \\ ${ }^{1}$ Navigation College, Dalian Maritime University, Dalian, 116026, Liaoning, P.R. China
}

\begin{abstract}
For the design of high-speed catamarans, different distances between slices have obvious interference with the total resistance of the catamaran. In order to accurately predict the hydrodynamic characteristics of the catamaran and explore the interference of the chip spacing on the resistance prediction, this paper uses a combination of CFD calculations and empirical formulas to predict the ship model resistance under different chip spacings and calculate them. The result is compared with the empirical formula. The results of the ship model test and the results calculated by the empirical formula were used to verify the numerical calculation results. The results show that the resistance change trend is consistent, and the numerical calculation method is effective and feasible. Finally, the numerical calculation method is compared with the ship model test method, and the result is within the error range, which has certain reference value for the design and optimization of the catamaran model parameters.
\end{abstract}

\section{Introduction}

With the development of the shipbuilding and ocean engineering fields and the improvement of the offshore equipment system, the research on high-performance ships has developed rapidly. My country has conducted long-term and multi-faceted research on catamarans, and has achieved many results in the development of catamarans. With the rapid development of computer technology, numerical calculation methods based on CFD have been widely used to analyze the resistance performance of catamarans. Domestically, Jiang Xueyun ${ }^{1}$ and others used COMPASS-WALCS_BASIC three-dimensional hydrodynamic calculation software to predict the wave load of small waterplane area catamarans with different chip spacings. Fang Jing ${ }^{2}$ and others designed an ultra-small unmanned catamaran and used OpenFOAM software to predict and analyze the hull resistance, providing a reference for the design of the ultra-small unmanned catamaran. Abroad, PK. Sahoo ${ }^{3}$ et al. based on the computational fluid dynamics (CFD) model and slender body theory, simulated the static water wave resistance characteristics of a Chinese-type cross-stern slender catamaran, and compared the CFD method to The predicted resistance is verified and compared with experimental data. Deniz Ozturk ${ }^{4}$ and others used CFD software to predict the full-scale resistance of a designed $15 \mathrm{~m}$ emergency response and rescue catamaran under wave conditions, and tested the seakeeping performance of this catamaran. This paper uses Siemens STAR_CCM+ software to solve the problem. This software is based on the finite volume method to solve the problem. It has a friendly interface, perfect grid function and powerful post-processing capabilities, which can be well applied to complex flow field calculations near ships. For high-speed catamarans, catamarans with different lamella spacing have different interference effects between the lamellas. In addition to the bow and stern shear wave interference that a catamaran has the same as an ordinary ship, the two hulls of the catamaran will also generate shear waves, scattered waves and reflected waves that will overlap each other. The overall resistance of the hull will produce greater or lesser interference ${ }^{5}$. If the setting of the sheet spacing can meet the favorable interference, the purpose of improving the resistance performance of the catamaran can be achieved. When the distance between the two plates is relatively close, the wave making interference between the two plates is obvious; when the distance between the two plates is relatively long, not only the interference of the plate wave making, but also the structural strength of the connecting bridge connecting the two plates should be considered. In order to meet the design requirements of the catamaran, it is necessary to select the appropriate sheet spacing. Therefore, this paper uses the combination of STAR_CCM+ software and empirical formulas to carry out the numerical calculation of the resistance performance of the high-speed catamaran, focusing on the analysis of the total resistance of the catamaran under different lamella spacing, and provides a reference for optimizing the span range of the connecting bridge.

\section{Empirical formulas and numerical methods}

In the past, in the preliminary design of the profile, the

\footnotetext{
"Corresponding author: ${ }^{\mathrm{b} z x f d m u @ d l m u . e d u . c n}$

aLiu_xiaopang@163.com, ${ }^{c} m y \_d m u @ 163 . c o m,{ }^{d}{ }_{W}$ lh1995@163.com
} 
empirical formula was used to make a rough estimate, and then the ship model test was carried out. Since the CFD numerical pool simulation, the hydrodynamic performance prediction at the initial design stage has been greatly improved, and the accuracy has been improved. It provides great help in the optimization of molding line, ship type, and structural strength.

For the prediction of catamaran resistance, Ma $\mathrm{Jian}^{6}$ proposed a method for forecasting the total resistance of a wave piercing catamaran in 2011. The total drag coefficient of the wave piercing catamaran is:

$$
C_{t}=C_{f}+C_{r}+\Delta C_{f}+C_{A P}
$$

Among them, the friction coefficient $C_{f}$ is based on the standard algorithm proposed by the 15 th ITTC in 1978:

$$
C_{f}=0.075 /(\lg \mathrm{Re}-2)^{2}
$$

The residual resistance $C_{r}$ of different sheet spacing is calculated by interpolation; rough subsidy $\Delta C_{f}$ is $0.4 \times 10^{-3}$; air resistance coefficient is $C_{A P}=(0.25 \sim 0.30) \times 10^{-3}$.

The total resistance of the wave piercing catamaran is: $R_{t}=0.5 \times C_{t} \times \rho \times S \times V_{s}^{2}$, where: $\rho$ is the density of the fluid, $\mathrm{kg} / \mathrm{m}^{3} ; S$ is the wet area of the hull, $\mathrm{m}^{2} ; V_{s}$ is the speed of catamaran, $\mathrm{m} / \mathrm{s}$.

CFD method is to use computer and discretization numerical simulation method to simulate and analyze fluid mechanics problems ${ }^{7-8}$. It solves mathematical equations that control fluid flow through a computer, and performs numerical simulation calculations on the motion of viscous or non-viscous fluids. The control equation used by the CFD software STAR_CCM+ is RANS (Reynolds-Averaged Navier-Stokes). The time average value is used to replace the statistical average value in the original equation. Assuming that the fluid is incompressible, the continuity equation and the RANS equation are as follows:

$$
\begin{gathered}
\frac{\partial \rho \vec{u}_{i}}{\partial x_{i}}=0 \\
\frac{\partial \rho \vec{u}_{i}}{\partial x_{i}}+\bar{u}_{j} \frac{\partial \vec{u}_{i}}{\partial x_{j}}+\frac{\partial\left(\vec{u}_{i} \vec{u}_{j}\right)}{\partial x_{i}}=f_{i}-\frac{1}{\rho} \frac{\partial \bar{P}}{\partial x_{i}}+v \frac{\partial^{2} \vec{u}_{i}}{\partial x_{i} \partial x_{j}}
\end{gathered}
$$

In the formula, $\rho$ is the fluid density; $v$ is the kinematic viscosity coefficient of the fluid; $f_{i}$ is the mass force experienced by the unit mass fluid; $\vec{u}_{i}$ and $\vec{u}_{j}$ represent the components of velocity in all directions; $\bar{P}$ is the time average value of pressure. The continuity equation (3) of the time-average flow and the RANS equation (4) together form the governing equations for incompressible fluid flow. Compared with the N-S equation, the RANS equation has more terms related to the pulsation, so the turbulence model is introduced, and the $\mathrm{k}-\varepsilon$ turbulence model is used in this paper.

As the ship's navigation speed increases, the ship's navigation state will change due to the different proportions of the flow support force. The Froude number $F r$ is usually used to express the relative speed of the ship.

$$
F r=V_{s} / \sqrt{g L}
$$

Where: $V_{s}$ is the ship's speed, $\mathrm{m} / \mathrm{s} ; g$ is the acceleration due to gravity, $9.81 \mathrm{~m} / \mathrm{s}^{2} ; L$ is the ship's length, $m$.

\section{Ship type parameters and test settings}

\subsection{Introduction to ship type}

The slice parameters of the high-speed catamaran model studied in this paper are as follows:

Table 1 Parameter Details of the slice

\begin{tabular}{|c|c|}
\hline Main scale & Value \\
\hline Length $L / m$ & 7.500 \\
\hline Beam $B / m$ & 1.025 \\
\hline Draft $d / m$ & 0.600 \\
\hline Displacement $\Delta / t$ & 2.502 \\
\hline
\end{tabular}

Use Soildworks software to draw the three-dimensional model of the ship. The three-dimensional model of the monolithic ship is shown in Figure 1:

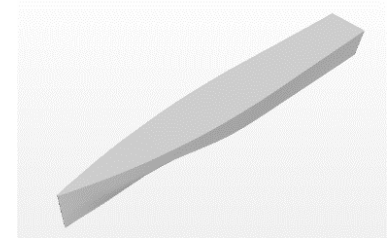

Figure 1 Hull geometries of the slice

\subsection{Related settings}

Set the calculation domain with length, width and height. The $\mathrm{X}$-axis of the reference coordinate system is positive when it points to the bow, the Y-axis is positive when it points to the port side of the hull, and the $\mathrm{Z}$-axis is positive upward. In the $\mathrm{X}$-axis direction, the length is 5Lpp (the bow is 1Lpp from the entrance and the stern is 3Lpp), in the Y-axis direction, the width is 4Lpp (the left and right sides of the ship are $2 \mathrm{Lpp}$ ), and the height is 3Lpp (the bottom of the ship is 2Lpp from the pool). The boundary of the calculation domain is set as: the inlet is designated as the velocity entrance; the outlet is designated as the pressure exit; side and side 2 on both sides of the domain are designated as the symmetry plane; Top and Bottom are designated as the velocity entrance; the ship surface is designated as the sliding wall.

The details are shown in Figure 2: 


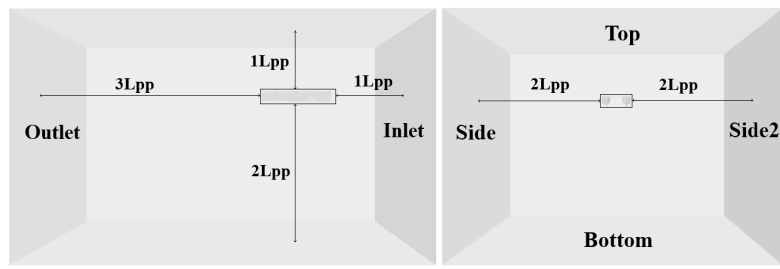

Figure 2 Calculational domain of the Catamaran

The computational domain is divided into grids. In order to reduce the grid, the grid size of the background domain is generally set to be large, and the grid is encrypted around the hull. The mesh of the free liquid surface is encrypted in the Z-axis direction, and the surface mesh of the ship is set. The main purpose is to set the thickness of the mesh to adjust the wall distance $\mathrm{y}+$ value. The meshing result is shown in Figure 3:

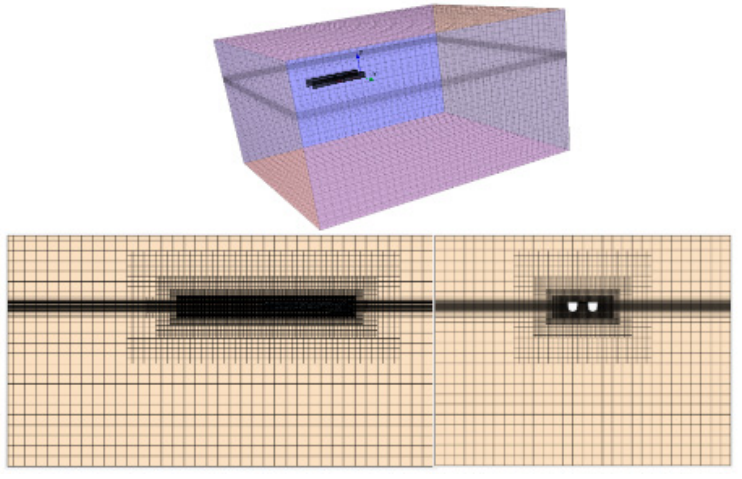

Figure 3 Illustration of mesh structure

\subsection{Numerical calculation results}

Using STAR_CCM+ software, the three-dimensional ship model was used to calculate the total resistance of 7 distances with different plate spacing ratios $k / \mathrm{b}=1.5 \sim 4.5$ when the speed was 2 20kn.

Select the situation when the speed of the high-speed ship is $16 \mathrm{kn}$, and the contour map of the numerical simulation wave making waveform of the monolithic hull and the hull distance $k / \mathrm{b}=1.5$ is shown in Fig. 4 .
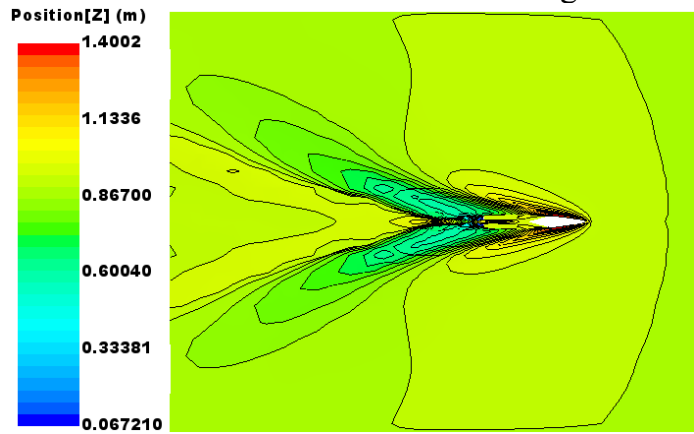

(a)

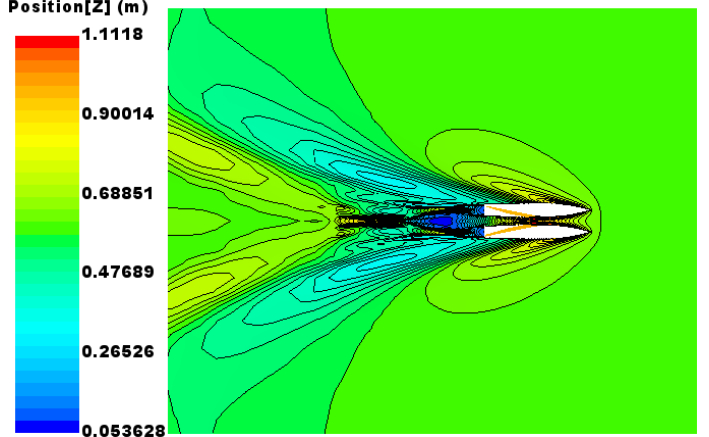

(b)

Figure 4 Wave contour map $($ speed $=16 \mathrm{kn})$

It can be seen that similar to the waves generated by the monolithic hull, the waves generated by the catamaran as a whole are more obvious, and the wave crests of the wake field are concentrated near the tail of the hull and spread outward. Unlike a monolithic hull, a catamaran will have a wave trough caused by the superposition of wakes, which is located on the centerline of the catamaran close to the starting position of the tail wave crest.

Comparing the difference of free surface wave making contours under different lamella spacings, select three working conditions with lamella distances $(k / \mathrm{b}=2$, $k / \mathrm{b}=3, k / \mathrm{b}=4)$. The contour map of free surface wave making under different working conditions is shown in Fig. 5.
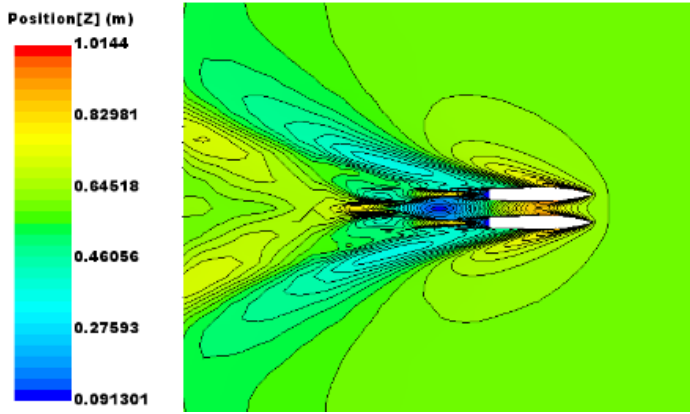

(a) $k / \mathrm{b}=2$

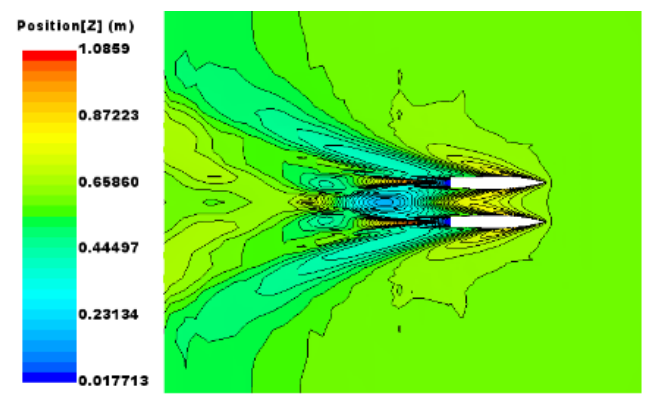

(b) $k / \mathrm{b}=3$ 

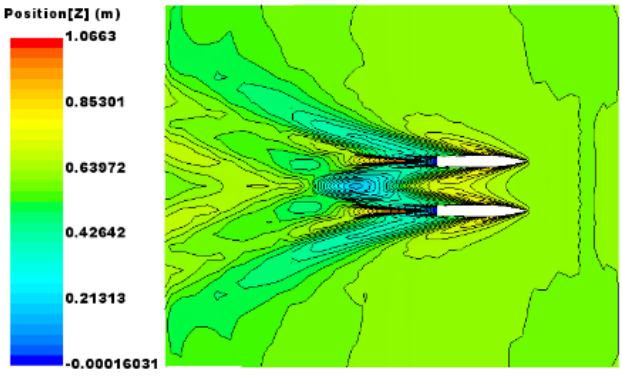

(c) $k / \mathrm{b}=4$

Figure 5 Free-surface wave making under different lamella spacing conditions

It can be seen from Figure 5 that the free-surface wave making waveforms under different sheet-to-body distance working conditions are significantly different. As the value of $k / \mathrm{b}$ increases, the wave making caused by the catamaran on the free surface of the flow field will increase. The inner side of the lamella (the side of the two adjacent lamellas of the catamaran) increases with the increase of $k / \mathrm{b}$, the wave making inside the lamella becomes more and more obvious, and the interference between the lamellas becomes more and more obvious. There are obvious crests and troughs. Especially in the stern part of the ship, there will be a deeper trough, and the position of the trough will also change depending on the size of $k / b$. These phenomena indicate the severe mutual interference of the water waves between the two hulls of the catamaran in the high-speed sailing state. The more obvious the wave making, the more obvious the impact on the total resistance of the hull.

\section{Analysis of calculation results}

Based on the setting of the logarithmic pool above, the total resistance of the catamaran in still water is predicted. Refer to the results of the tank drag test of A.F.Molland ${ }^{9}$, etc., Insel ${ }^{10}$, etc., the drag coefficient is dimensionless according to $C_{i}=F_{i} /\left(0.5 \rho V^{2} S\right)$. When $k / \mathrm{b}=3.5$ is selected, the total resistance coefficient obtained from the numerical simulation calculation results is shown in Table 2.

Table 2 Simulation calculation results

\begin{tabular}{|c|c|c|c|}
\hline Fr & Pool test value & $\begin{array}{c}\text { Simulation } \\
\text { calculation value }\end{array}$ & $\begin{array}{c}\text { Relative error } \\
/ \%\end{array}$ \\
\hline 0.2 & 6.9016 & 7.388997 & 7.062086 \\
\hline 0.4 & 9.918 & 10.09267 & 1.761098 \\
\hline 0.5 & 14.0164 & 12.93668 & -7.70323 \\
\hline 0.6 & 12.16314 & 12.39563 & 1.911362 \\
\hline 0.8 & 8.8689 & 8.170515 & -7.87454 \\
\hline 0.9 & 7.8852 & 6.950739 & -11.8508 \\
\hline 1 & 7.3279 & 6.154461 & -16.0133 \\
\hline 1.1 & 6.796044 & 5.74029 & -15.5348 \\
\hline
\end{tabular}

The numerical calculation results are compared with the physical tank test results, considering that the uncertain factors of the scale effect will affect the conversion results, but the overall error is less than $20 \%$. Therefore, the CFD numerical calculation method applied to predict the total resistance of the catamaran is reasonable, feasible and have higher accuracy.

When $k / b=3.5$, the variation curve of ship's total resistance with Froude number $F r$ obtained by empirical formula and numerical simulation is shown in Figure 6.

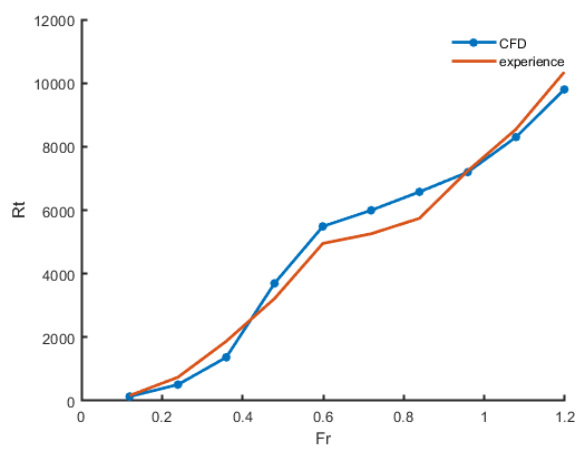

Figure 6 Change curve of total drag with $\mathrm{Fr}$

It can be seen from Figure 6 that for the change of total resistance, the CFD calculation results are consistent with the growth trend of the empirical formula calculation results. Therefore, it is feasible to use the numerical simulation method to predict the resistance change of the catamaran under different lamella spacing.

Taking into account the influence factors of different chip spacing on the resistance, $k / \mathrm{b}=1.5,2.0,2.5,3.0,3.5$, $4.0,4.5$ were selected respectively for comparative analysis, and the total resistance $R_{t}$ and the total resistance coefficient $C_{t}$ obtained were proportional to the Frude number $\mathrm{Fr}$. The change curve of $R_{t}$ and $C_{t}$ is shown in Figure 7 and Figure 8.

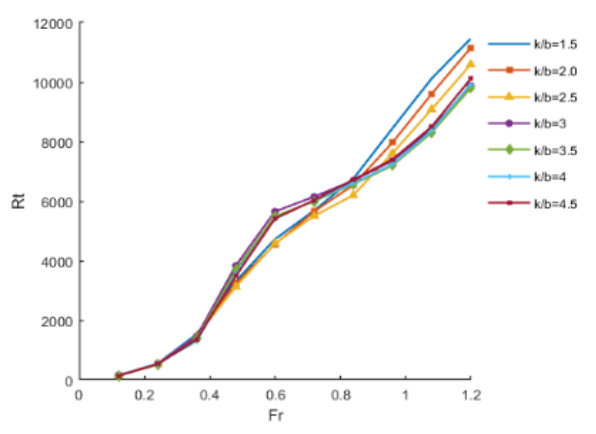

Figure 7 Variation of total resistance with $F r$ under different sheet spacing

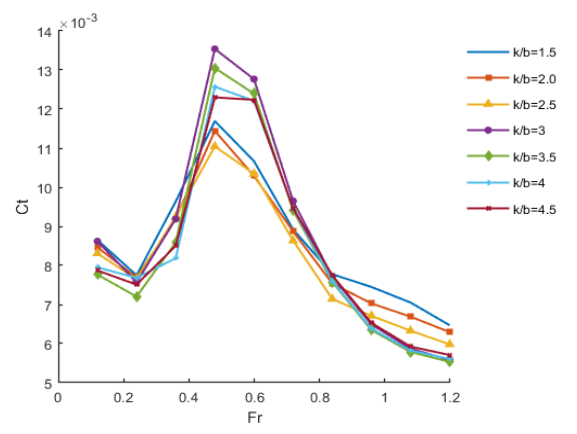

Figure 8 Variation of with total drag coefficient $F r$ under different sheet spacing 
It can be seen from Figure 7 that as the ship's speed increases, the total resistance of the hull continues to increase. When the Froude number $F r$ is about 0.6, the total resistance of the catamaran has a slower growth trend, and the Froude number When $F r$ is $0.8 \sim 1.0$, the growth trend of total resistance becomes steeper. It can be seen from Figure 8 that when the Froude number $\mathrm{Fr}$ is about 0.5 , the wave making interference of the catamaran is the most serious, with a peak. After this peak, the wave-making resistance of the catamaran decreases as the speed increases.

Further analysis of the obtained results shows that under a certain Froude number different resistance coefficients change with the distance $k / \mathrm{b}$ of the sheet. Select $F r=0.2,0.4,0.5,0.6,0.8,1.1$ for low speed, medium speed, and high speed respectively. Obtain the variation of the drag coefficient with the distance between the sheets, as shown in Figure 9.

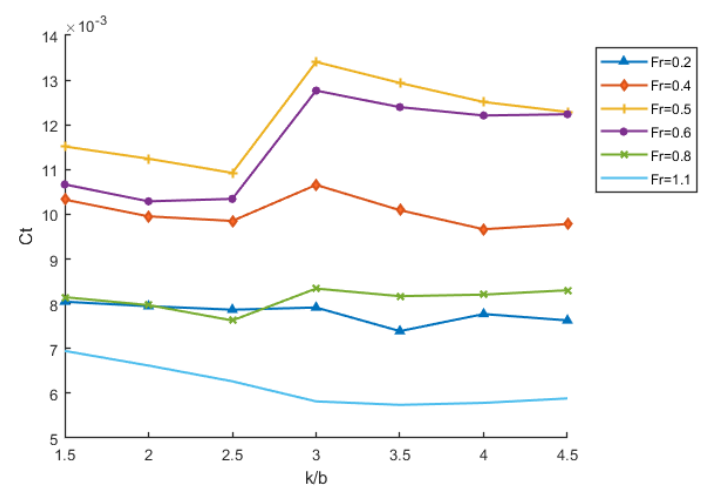

Figure 9 The total drag coefficient varies with the distance between the sheets

It can be seen from Figure 9 that for catamarans under low-speed and high-speed navigation, the interference of wave-making resistance is smaller than that of medium-speed navigation (when the Frude number $\mathrm{Fr}$ is about 0.5 ).

Comparing the total resistance curves of ships under different $k / \mathrm{b}$, when $F r=0.2, k / \mathrm{b}=3.5$, there is a significant reduction in the wave-making interference of the catamaran; And when $F r$ is between 0.4 and 0.8; When $k / \mathrm{b}=2.5$, there will be a significant decrease; when $F r=1.1$, it will gradually decrease when $k / \mathrm{b}=3$. Considering the practical application of catamarans, it is recommended to use $k / \mathrm{b}=3$ in the design of high-speed catamarans, while $k / \mathrm{b}$ of 2.0 to 3.0 is more appropriate for medium-speed catamarans. Experiments have proved that the interference of wave making resistance on catamarans is closely related to the distance between the plates and the speed.

\section{Conclusion}

This paper adopts a real high-speed catamaran ship, uses CFD simulation software to quantify the resistance of the ship and compares and analyzes it. The simulation calculation is carried out by setting different chip spacings and different speed conditions. The simulation results were compared with the results calculated by the empirical formula and the ship model test results given in the references. The results show that the CFD numerical calculation method is reasonable and feasible, the resistance prediction accuracy is high, and the calculation speed is fast.

The wave-making resistance interference of high-speed catamarans is closely related to the distance between the plates and the speed. In the design process of the catamaran, the setting of the distance between the lamellas needs to be adjusted in consideration of practical scenarios, and the determination of the lamella interference coefficient is of great significance to the design of the actual ship. There are many factors that affect the wave resistance between the plates. This article only conducts an in-depth study on the two factors of plate spacing and speed. Further research is needed on the influencing factors such as prismatic coefficient and draught.

\section{References}

1. Jiang Xueyun, Feng Guoqing, Zhang Qingyue, Ren Huilong. (2016) The effect of plate spacing on the lateral wave load of SWATH ship [J]. Ship Science and Technology, 38(03): 13-17.

2. Fang Jing, Huang Jing, Feng Baiwei, Chang Haichao, Li Zilu. (2018) The overall design of ultra-small catamaran unmanned ship based on CFD[J]. Ship Engineering, 40(05): 1-3+56.

3. Sahoo P, Doctors L, Pretlove L. CFD prediction of the wave resistance of a catamaran with staggered demihulls. 2006.

4. zturk Deniz, Delen Cihad, Mancini Simone, Serifoglu Mehmet Ozan, Hizarci Turgay. (2021) Full-Scale CFD Analysis of Double-M Craft Seakeeping Performance in Regular Head Waves[J]. Journal of Marine Science and Engineering, 9(5).

5. Du Youwei, Zhou Xinyuan.(2019) Resistance analysis and ship optimization of wave piercing catamaran[J]. Journal of Wuhan Institute of Shipbuilding Technology, 2019(1):87-90.

6. Ma Jian, Zhang Zaifu, Li Huimin. (2011)Analysis of influencing factors of residual resistance of wave piercing catamaran and total resistance prediction method[J]. Ship Mechanics, 15(Z1): 32-39.

7. Zha Ruosi. Numerical analysis of wave resistance increase of monohull and catamaran[D]. Shanghai Jiaotong University, 2015.

8. Liu Jinling. Research on motion simulation and attitude control of wave piercing catamaran[D]. Harbin Engineering University, 2013.

9. Molland A F, Wellicome J F, Couser P. Resistance Experiments on a Systematic Series of High-Speed Displacement Catamaran Forms: Variations of Length-Displacement Ratio and Breadth-Draugh Ratio. 1996.

10. Insel M, Molland A F. (1992) An investigation into the resistance components of high speed displacement catamarans[J]. transactions of the royal institution of naval architects, 1992. 